\title{
Le ricerche dirette nella diagnosi di Toxoplasmosi. Esperienza di un triennio
}

\author{
Alessandra Orsi \\ Laboratorio di Microbiologia e Virologia - Azienda Sanitaria Careggi Firenze
}

Use of direct techniques in toxoplasmosis diagnosis. Three years experience.

\section{SUMMARY}

Since serologic tests have not proven useful for diagnosis of toxoplasmosis in immunodeficient patiens (patients with hematologic malignancies, bone marrow transplant, solid organ transplant or AIDS) and sometimes in immunocompetent patients (to establish congenital infection in the fetus and newborn and ocular toxoplasmosis) we have used the polymerase chain reaction (PCR) to detect Toxoplasma gondii DNA and tissue cell cultures to isolate the parasite. DNA was amplified using external primers and, in a second step, internal primers, in a nested PCR system.

The isolation of parasite was performed on fibroblast cell culture (MRC-5). I 30 samples were examinated:amniotic fluid (53), cerebrospinal fluid (CSF) (37), blood (32),aqueous fluid (3),biopsy (3), urine (I) and sample from abortion(I).

The PCR was performed on all samples and was positive in eight cases: three amniotic fluids, two CSF, two blood and one biopsy. The cell culture was made on 63 samples (53 amniotic fluid and 10 blood) and was positive in two cases of amniotic fluid confirming the PCR results. Because positive predictive value of PCR range from 50 $\%$ to $80 \%$ this technique should not be used alone for prenatal diagnosis of congenital toxoplasmosis. The positive samples other than amniotic fluid came all from AIDS patients.

\section{INTRODUZIONE}

La toxoplasmosi è una malattia causata da un protozoo parassita intracellulare obbligato il Toxoplasma gondii.

Il $T$. gondii è ampiamente diffuso tra la popolazione mondiale infatti si calcola che circa il $70 \%$ degli individui è infettato dal parassita tuttavia solo il $10-20 \%$ dei soggetti infetti presenta sintomatologia. Le principali manifestazioni cliniche sono linfoadenopatia, corioretinite e meno frequentemente miocardite.

Nei pazienti immunocompromessi la toxoplasmosi è una malattia grave e spesso fatale e si verifica in genere per riattivazione di un'infezione latente. Pazienti a rischio di toxoplasmosi sono quelli con tumori ematologici, soprattutto linfoma, pazienti sottoposti a trapianto di midollo osseo o di organi solidi e pazienti affetti da Aids. Nei pazienti immunocompromessi le manifestazioni cliniche sono principalmente rappresentate da encefalite, polmonite, miocardite e da infezioni oculari.

La diagnosi di toxoplasmosi viene in genere eseguita mediante ricerche sierologiche che mettono in evidenza anticorpi IgG, IgM, IgA e IgG a bassa avidità.

Le indagini indirette non sono risolutive per la diagnosi di infezione in pazienti immunodepressi o per eseguire la diagnosi prenatale in donne gravide in cui sia stata rilevata una infezione attiva da toxoplasma o per definire un'infezione conge- nita nel neonato o ancora nelle infezioni di pazienti trapiantati o in infezioni oculari (2).

In questi casi si rende necessario l'uso di ricerche dirette basate sulla dimostrazione del parassita o di sue parti (1).

Circa tre anni fa nel nostro Laboratorio è stata iniziata la ricerca del T.gondii con metodi diretti. Scopo del presente lavoro è di riferire sulla esperienza di questi anni.

\section{MATERIALI E METODI}

Campioni. Sono stati esaminati 130 campioni biologici provenienti da 129 pazienti ricoverati in ospedali dell'area fiorentina o ambulatoriali. I pazienti erano rappresentati soprattutto da donne gravide (53) alle quali era stata diagnosticata una infezione acuta da $T$. gondii e che si sottoponevano alla diagnosi prenatale. Gli altri erano rappresentati da pazienti immunodepressi HIV positivi (62), neonati con sospetto di toxoplasmosi congenita (11), pazienti con infezione oculare (3) e da una paziente con aborto spontaneo. I campioni erano costituiti da: liquido amniotico 53 , liquido cefalo-rachidiano 37 , sangue 32 , umor acqueo 3 , biopsia cerebellare 1 , biopsia linfonodale 2 , materiale abortivo 1 , urina 1 .

L'isolamento colturale è stato eseguito su 63 campioni (tutti i liquidi amniotici, 10 campioni di sangue, 2 biopsie, 1 urina).

Isolamento di T. gondii in coltura cellulare. Sono stati utilizzati fibroblasti da polmone di embrione umano (MRC-5 Vircel - Amplimedical) coltivati 
in monostrato su un vetrino contenuto in una provetta (tecnica degli shell vials). I campioni sono stati seminati negli shell vials ed incubati a $37^{\circ} \mathrm{C}$ in atmosfera di $\mathrm{CO}_{2}$ al 5\% per 10 giorni.

I campioni di urina, di liquido amniotico e di liquido cefalorachidiano sono stati centrifugati a 2000 rpm per 10 minuti.

Il pellet ottenuto è stato lavato per due volte con PBS e quindi inoculato in sei shell vials in quantità di 200 microlitri per shell vial. Dai campioni di sangue sono stati separati i leucociti con destrano al 6\% (Argene - Amplimedical) ed il buffy coat ottenuto lavato per due volte con PBS e seminato in sei shell vials in quantità di 200 microlitri per shell vial.

Le cellule ottenute dai campioni bioptici dopo separazione meccanica sono state centrifugate, lavate e seminate come gli altri materiali.

Il vetrino è stato esaminato per il rilievo di tachizoiti sia mediante colorazione di Giemsa (figura I) sia mediante immunofluorescenza indiretta con anticorpi monoclonali (Toxoplasma gondii 30KD (clone G2-9 Argene-Amplimedical) (figura II) dopo tre giorni e dopo dieci giorni di incubazione. Rilievo del DNA di $T$. gondii mediante PCR. I pellet ottenuti dai vari materiali biologici e il buffy coat ottenuto dai campioni ematici sono stati sottoposti ad estrazione del DNA mediante lisi con alcali a caldo (EXTRAcell Amplimedical); il DNA estratto è stato amplificato con tecnica di PCR di tipo "nested" utilizzando due paia di primer per il gene B1 del T.gondii ottenendo un primo amplificato di 193 paia di basi ed un secondo amplificato interno di 88 paia di basi. La sensibilità del kit utilzzato è di 10 genomi / reazione. (Toxoplasma gondii oligomix Amplimedical).

DNA di tachizoiti di T.gondii è stato utilizzato come controllo positivo ed acqua ultrapura come controllo negativo.

L'amplificazione è stata eseguita in un thermal cycler Hybaid Express.

Aliquote dei prodotti di amplificazione sono state analizzate mediante elettroforesi su gel di agarosio contenente bromuro di etidio e visualizzate a luce UV. Le dimensioni del DNA di $T$. gondii corrispondono ad una banda di 88 pb (figura III).

\section{RISULTATI}

La ricerca del DNA eseguita su 130 campioni ha dato esito positivo in otto campioni: tre campioni di liquido amniotico, due di liquido cefalo-rachidiano, due campioni di sangue, una biopsia linfonodale. I campioni positivi di liquor, sangue e la biopsia provenivano da pazienti HIV positivi. I campioni di liquido amniotico invece provenivano da donne gravide nelle quali era stata diagnosticata infezione acuta da T. gondii. L'amniocentesi era stata eseguita dopo la diciotte-

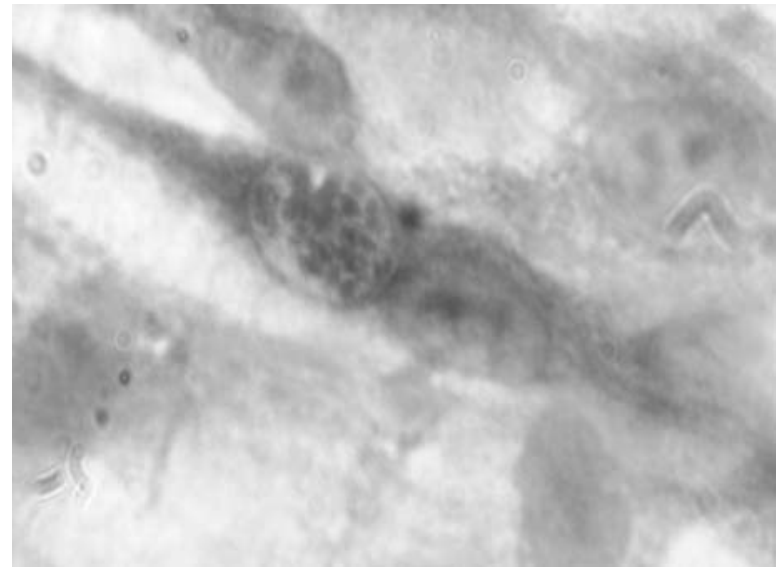

Figura I - Toxoplasma gondii: isolamento da liquido amniotico in coltura di MRC-5; colorazione di Giemsa.

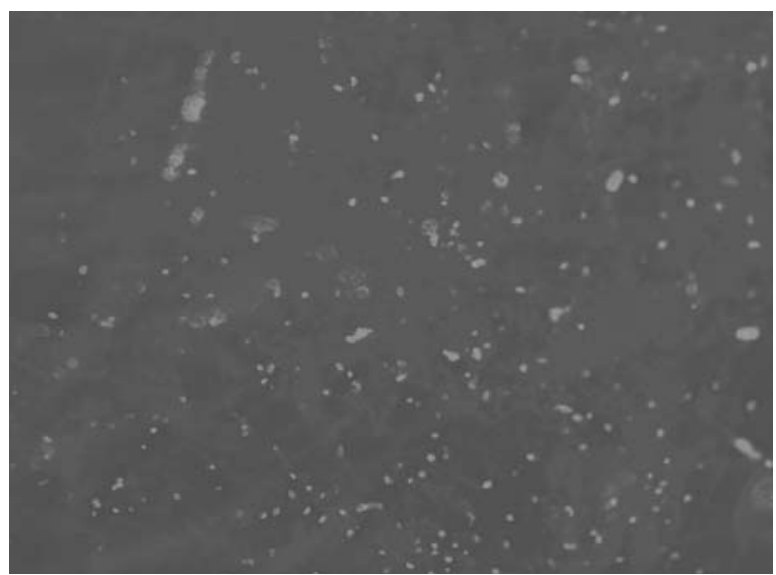

Figura II - Toxoplasma gondii:

isolamento da liquido amniotico; colorazione con IF.

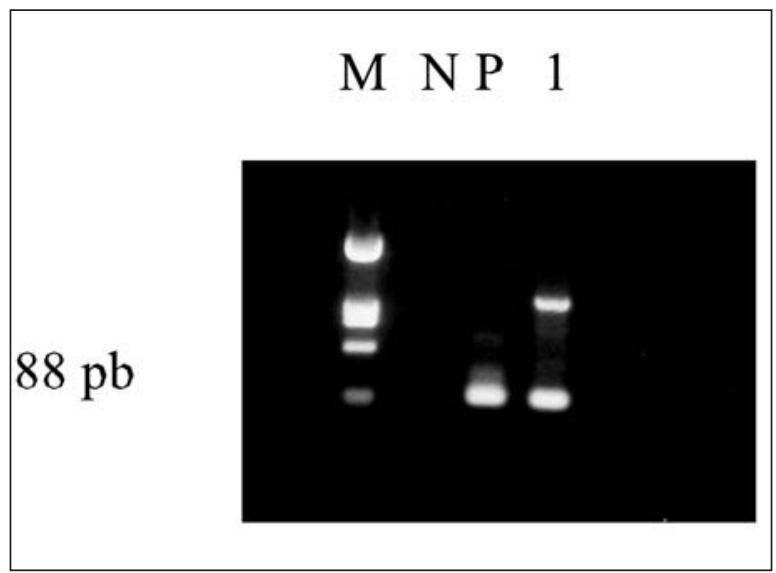

Figura III - Toxoplasma gondii:

DNA elettroforesi su gel di agarosio dopo PCR

$M$ marker $N$ negativo $P$ positivo I liquido amniotico

sima settimana di gestazione.

Dei campioni di liquido amniotico con PCR positiva sono risultati positivi alla coltura solo due.

Sugli altri campioni positivi alla PCR non è stato eseguito l'isolamento colturale.

Tutti i campioni con PCR negativa sui quali è 
stato eseguito l'esame colturale sono risultati negativi.

\section{CONCLUSIONI}

Nei pazienti immunodeficienti in cui si sospetti toxoplasmosi le indagini sierologiche non sono di nessun aiuto. Per stabilire la diagnosi in questi soggetti si raccomanda di eseguire indagini dirette (8) consistenti nel rilievo del DNA del T.gondii dal sangue o da altri materiali biologici infetti e/o nell'isolamento del parassita dagli stessi materiali.

Qualora si sospetti neurotoxoplasmosi raramente il parassita viene isolato dal liquido cefalorachidiano e pertanto si procede solo con il rilievo di DNA con PCR $(7,9)$.

La tecnica di PCR ha consentito di eseguire diagnosi di toxoplasmosi in quattro pazienti HIV positivi in due dei quali a livello cerebrale.

Nei pazienti immunocompetenti la toxoplasmosi viene sempre diagnosticata con indagini sierologiche. In certi casi le ricerche anticorpali non sono risolutive ad esempio:

- quando si voglia stabilire se il feto di una donna con infezione acuta da T.gondii sia stato o no infettato cioè quando si voglia eseguire diagnosi prenatale

- quando si voglia stabilire se l'infezione nel neonato è congenita

- quando si sospetti che il parassita sia responsabile di una infezione oculare

In tali situazioni si può ricorrere ad indagini dirette.

La diagnosi prenatale viene eseguita mediante ricerca del DNA con PCR sul liquido amniotico prelevato non prima della $18^{\text {a }}$ settimana di gestazione. La PCR è la tecnica diretta più sensibile $\mathrm{e}$ più rapida e pertanto è stata eseguita su tutti i campioni, tuttavia poiché secondo i dati della letteratura $(7,10-12)$ un risultato positivo di PCR nel liquido amniotico ha un valore predittivo positivo (VPP) variabile dal $50 \%$ al $80 \%$ la PCR deve essere eseguita sempre insieme all'isolamento del parassita che assume in tal caso una grande importanza per stabilire l'infezione del feto. Sui tre campioni di liquido amniotico positivi alla PCR infatti solo in due è stato isolato il parassita. La diagnosi prenatale dovrebbe essere eseguita prima possibile poiché il trattamento della madre può ridurre il danno fetale.

Per stabilire l'infezione congenita nel neonato la ricerca di IgM e di IgA non è sempre interpretabile e sono stati inoltre segnalati casi di sieronegatività transitoria (6) pertanto devono essere utilizzate ricerche tendenti all' isolamento colturale del parassita e alla dimostrazione del suo DNA (10). Tali ricerche possono essere eseguite su sangue, liquido cefalorachidiano e su urina (4) materiale di più facile reperibilità soprattutto nei neonati (5).

Infine per quanto riguarda le infezioni oculari non sono sempre diagnosticabili solo su base clinica, gli anticorpi specifici sono presenti nel siero spesso a basso titolo e pertanto la ricerca del DNA nel parassita a livello locale può essere risolutiva (3).

\section{RINGRAZIAMENTI}

Desidero ringraziare per la fattiva e attenta collaborazione i Tecnici di laboratorio signore Massai Miriam e Stefanelli Marta.

\section{BIBLIOGRAFIA}

1. Bastien P. Molecular diagnosis of toxoplasmosis. Trans R Soc Trop Med Hyg 2002 ; 96 Suppl S205-15.

2. Botterel F, Ichai P, Feray C, et al. Disseminated toxoplasmosis,resulting from infection of allograft,after orthotopic liver transplantation usefulness of quantitative PCR. J Clin microbiol 2002; 40: 1648-50.

3. Bou G, Figueroa MS, Martì-Belda P, Navas E, Guerrero A. Value of PCR for detection of Toxoplasma gondii in aqueous humor and blood samples from immunocompetent patients with ocular Toxoplasmosis. J Clin Microbiol 1999; 37: 3465-8.

4. Fuentes I, Rodriguez M, Domingo CJ, Del castillo F, Juncosa T, Alvar J. Urine sample used for congenital Toxoplasmosis diagnosis by PCR. J Clin Microbiol 1996; 10: 2368-71.

5. Gratzl R, Hayde M, Kohlhauser C, et al. Follow-up of infants with congenital toxoplasmosis detected by plymerase chain reaction analysis of amniotic fluid. Eur J clin Microbiol Infect Dis 1998; 17 (12): 853-8.

6. Jaisson-Hot I, Wallon M, al Kurdi M, et al. Congenital toxopasmosis. Transitory negative serology. Press med 2001; 30: 1001-4.

7. Julander I, Martin C, Lappalainen M, Guy E, Isberg B, Evengar B. Polymerase chain reaction for diagnosisi of cerebral toxoplasmosis in cerebrospinal fluid in HIV-positive patients. Scand J Infect Dis 2001; 33 (7): 538-41.

8. Montoya JG. Laboratory diagnosis of Toxoplasma gondii infection and Toxoplasmosis. The $\mathrm{J}$ of Infec Dis 2002; 185 Suppl 1: S73-82.

9. Parmley SF, Goebel FD, Remington JS. Detection of Toxoplasma gondii in cerebrospinal fluid from AIDS patients by polymerase chain reaction. J Clin Microbiol 1992; 30: 3000-2.

10. Robert-Gangneux F, Gavinet MF, Ancelle T, Raymond J, Tourte-Schaefer C, Dupouy-Camet J. Value of prenatal diagnosis, early postnatal diagnosis of congenital Toxoplasmosis: retrospective study of 110 cases. J Clin Microbiol 1999; 37 (9): 2893-8.

11. Romand S, Wallon M, Franck J, Thulliez P, Peyron F, Dumon H. Prenatal diagnosis using polymerase chain reaction on amniotic fluid for congenital Toxoplasmosis. Obstet Gynecol 2001; 97: 296-300.

12. Vidigal PV, Santos DV, Castro FC, Couto FJ, Vitor RW, Brasileiro Filho G. Prenatal toxoplasmosis dia- 
gnosis from amniotic fluid by PCR. Revista da Sociedade Brasileira de Medicina Tropical 2002; 35 (1): 1-6.

\author{
Alessandra Orsi \\ Laboratorio Microbiologia e Virologia \\ Azienda Ospedaliera Careggi \\ Viale Morgagni 85 \\ 50134 Firenze \\ Tel.055 4279288 ; fax 0554279830 \\ E-mail: labmicro12@a0-careggi.toscana.it
}

